\title{
On the combinatorial structure of arrangements of oriented pseudocircles
}

\author{
Johann Linhart \\ Institut für Mathematik, Universität Salzburg, \\ Hellbrunner Straße 34, 5020-Salzburg, Austria \\ johann.linhart@sbg.ac.at
}

Ronald Ortner

Department Mathematik und Informationstechnologie, Montanuniversität Leoben, Franz-Josef-Straße 18, 8700-Leoben, Austria

ronald.ortner@unileoben.ac.at

Submitted: Feb 27, 2004; Accepted: Apr 5, 2004; Published: Apr 13, 2004

MR Subject Classifications: 52C30, 52C40

\begin{abstract}
We introduce intersection schemes (a generalization of uniform oriented matroids of rank 3) to describe the combinatorial properties of arrangements of pseudocircles in the plane and on closed orientable surfaces. Similar to the FolkmanLawrence topological representation theorem for oriented matroids we show that there is a one-to-one correspondence between intersection schemes and equivalence classes of arrangements of pseudocircles. Furthermore, we consider arrangements where the pseudocircles separate the surface into two components. For these strict arrangements there is a one-to-one correspondence to a quite natural subclass of consistent intersection schemes.
\end{abstract}

\section{Introduction}

The Folkman-Lawrence topological representation theorem for oriented matroids states that each oriented matroid may be represented by an oriented pseudosphere arrangement. Bokowski (cf. [4] where further references are given) gave an axiomatization of oriented matroids based on so-called hyperline sequences. This alternative axiomatization is especially natural in the case of rank 3 and led to a new direct proof of the Folkman-Lawrence topological representation theorem in this special case [5] and for arbitrary rank [6].

In this paper, we give a generalization of rank 3 uniform oriented matroids (with respect to Bokowski's axiomatization) called intersection schemes that describe simple 
arrangements of oriented (pseudo)circles just as uniform oriented matroids of rank 3 describe simple arrangements of (pseudo)lines. Since intersection schemes may also describe arrangements of pseudocircles on the torus and in general on closed orientable surfaces, we aim to show that each intersection scheme may be represented by an arrangement of oriented pseudocircles on some closed orientable surface. Since pseudocircles on surfaces such as the torus need not separate the surface into two parts, we also consider the question, in which cases an intersection scheme has a topological representation as an arrangement of separating pseudocircles. Here one can find a quite natural subclass of consistent intersection schemes for which a one-to-one correspondence to the mentioned strict arrangements holds.

\section{Preliminaries}

By a pseudocircle we mean an oriented closed Jordan curve in the plane or on some closed orientable surface. For the latter we assume it has a fixed orientation, no matter which one, while the plane is assumed to be oriented in the usual way.

A pseudocircle $\gamma$ is called separating, if its complement consists of two connected components. With regard to one of these $\gamma$ is oriented counterclockwise. This component is called the interior of $\gamma$. In the plane we assume all pseudocircles to be oriented counterclockwise, so that the interior is the bounded component.

Definition 2.1. An arrangement of pseudocircles is a finite set $\Gamma=\left\{\gamma_{1}, \ldots, \gamma_{n}\right\}$ of oriented closed Jordan curves in the plane or on a closed orientable surface such that

(i) no three curves meet each other at the same point,

(ii) if two pseudocircles have a point in common, they cross each other in that point.

(iii) $\left|\gamma_{i} \cap \gamma_{j}\right|<\infty$ for all $\gamma_{i}, \gamma_{j} \in \Gamma$,

(iv) $\bigcup_{i=1}^{n} \gamma_{i}$ is connected.

An arrangement of separating pseudocircles is called strict.

Given an arrangement $\Gamma$ of two or more pseudocircles, we may consider the intersection points of the pseudocircles as vertices and the curves between the intersections as edges. Thus we obtain in a natural way an embedding of a (multi)graph which we call the arrangement graph of $\Gamma$. Arrangement graphs can be characterized using the following coloring algorithm for embeddings of 4-regular graphs due to C. Iwamoto and G.T. Toussaint [13].

1. Color an arbitrary yet uncolored edge with a new color.

2. If there are four edges $e_{1}, e_{2}, e_{3}, e_{4}$ such that

- $e_{1}, e_{2}, e_{3}, e_{4}$ are incident with the same vertex, 
- $e_{1}, e_{2}, e_{3}, e_{4}$ are in clockwise order,

- $e_{1}$ is colored with color $i$ and

- $e_{3}$ is not yet colored,

then color $e_{3}$ with color $i$.

3. Repeat step 2 until there is no quadruple of edges satisfying the condition.

4. Repeat steps $1-3$ until all edges are colored.

Having colored an embedding of an arbitrary 4-regular graph $G=(V, E)$ with colors $1, \ldots, c$, one obtains a partition of the graph's edge set $E=E_{1} \cup \ldots \cup E_{c}$. Adding the corresponding incident vertices to the edge sets yields $c$ subgraphs of $G$.

Proposition 2.2. A 4-regular graph $G=(V, E)$ is the arrangement graph of an arrangement of pseudocircles if and only if there is an embedding of $G$ such that all subgraphs arising from the algorithm described above are 2-regular.

Proof. Straightforward.

For the sake of simplicity in what follows we confine ourselves mostly to 2-admissible arrangements, which means that each two pseudocircles shall cut each other in at most two points. However, all results extend to general arrangements of pseudocircles straightforwardly (cf. the remarks after Definition 2.5 below).

We may describe an arrangement of (labelled) pseudocircles $\left\{\gamma_{1}, \gamma_{2}, \ldots, \gamma_{n}\right\}$ as follows. Consider a counterclockwise walk on each pseudocircle beginning in an arbitrary vertex. Whenever we meet a vertex we note the index $k$ of the curve $\gamma_{k}$ we cross provided with a sign that indicates whether $\gamma_{k}$ comes from the left $(+)$ or from the right $(-) .{ }^{1}$ Thus we obtain for each pseudocircle a cyclic list. For the arrangement in Figure 1 these lists look like this: $((+2,-2,+3,+4,-4,-3),(+3,-3,+1,-1,+4,-4),(+4,-4,+1,-1,+2,-2)$, $(+1,-1,-3,+2,-2,+3))$. Each vertex is represented by exactly two entries in this scheme, which always have different sign. The arising tuple of cyclically ordered lists is called the intersection scheme of the arrangement. In the case of non-strict arrangements and arrangements where two pseudocircles cut each other in more than two points it may be necessary to provide entries with an additional index in order to identify entries corresponding to the same vertex.

We introduce the notion of crossings. By a crossing we mean a pair $\left(\gamma_{j}, v\right)$, where $\gamma_{j}$ is a pseudocircle in the arrangement and $v$ is a vertex lying on $\gamma_{j}$. If $\gamma_{k}$ is the other pseudocircle containing $v$, the crossing $\left(\gamma_{j}, v\right)$ will be denoted by $(j,+k)$ or $(j,-k)$, respectively, where the sign has the meaning explained above. Evidently, the crossing $(j, \pm k)$ is represented by the entry $\pm k$ in the $j$-th list. The corresponding vertex will be denoted by $v(j, \pm k)$, so that $v(j, \pm k)=v(k, \mp j)$.

\footnotetext{
${ }^{1}$ For separating curves $\gamma_{k}$ '+' means that we enter the interior, while '-' indicates that we leave the interior.
} 


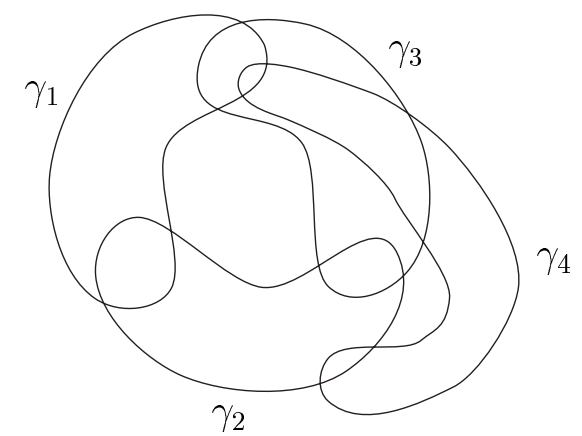

Figure 1: An arrangement of pseudocircles.

The edges in the arrangement graph correspond to the pairs of neighbored crossings in any list of the scheme (note that due to the cyclical nature of the lists the last and the first crossing in the same list are neighbors as well). Hence, we may define a walk in an intersection scheme as follows.

Definition 2.3. A walk in an intersection scheme $A$ of an arrangement is a finite sequence of crossings $\varepsilon_{1}, \ldots, \varepsilon_{m}$ such that two successive crossings $\varepsilon_{i}, \varepsilon_{i+1}$ are either neighbored in a list or correspond to the same vertex. A closed walk in $A$ is a walk $\varepsilon_{1}, \ldots, \varepsilon_{m}$ where either $\varepsilon_{1}=\varepsilon_{m}$ or $\varepsilon_{1}, \varepsilon_{m}$ correspond to the same vertex.

Now we are able to give a definition of (abstract) intersection schemes.

Definition 2.4. An (abstract) intersection scheme $A$ is an $n$-tuple of cyclically ordered lists such that:

(i) The $i$-th list of $A$ consists of elements $\in\{+1,-1,+2,-2, \ldots,+n,-n\} \backslash\{+i,-i\}$.

(ii) Entries do not occur more than once in a list.

(iii) The entry $+k$ occurs in list $i$ if and only if the entry $-i$ occurs in list $k$.

The definitions of crossing and walk extend naturally to $n$-tuples of cyclically ordered lists satisfying (i) - (iii). Thus the following condition makes sense.

(iv) For each two crossings there is a walk connecting them.

Definition 2.5. An intersection scheme $A$ is said to be pure if furthermore

(v) the entry $+k$ occurs in a list if and only if $-k$ does.

As mentioned above, for arrangements in general one needs additional indices for identification of entries corresponding to the same vertex. These generalized intersection schemes can be defined analogously to Definition 2.4 above, where each entry in (i) is provided with an additional index $\ell$, written $\pm k^{(\ell)}$. Moreover, a generalized intersection 
scheme is pure if in each list the entries of the form $\pm k^{(\ell)}$ with fixed $k$ (read cyclically) have alternating sign. In what follows we will consider mostly "ordinary" intersection schemes. However, all results can easily be extended to the case of generalized intersection schemes.

We call an intersection scheme $A$ (strictly) representable if there is a (strict) arrangement $\Gamma$ of pseudocircles with intersection scheme $A$. $\Gamma$ then is called a (strict) topological representation of $A$. The reader can easily verify that intersection schemes of arrangements of pseudocircles satisfy all conditions of Definition 2.4. Moreover, strict arrangements always have pure intersection schemes.

Arrangements of pseudocircles naturally arise in many problems of combinatorial and computational geometry. On the one hand, many well-known results concerning arrangements of lines can be generalized to arrangements of pseudocircles (see e.g. [7], [8], [19], [18]). On the other hand, there are many applications to other geometrical problems such as motion planning (see e.g. [1], [12], [14]) or Venn diagrams ([11]). For other applications see e.g. [1], [7] and [8]. Our approach here is closely related to oriented matroids and their connection with arrangements of pseudolines and special classes of arrangements of pseudocircles (cf. [4], p.569 or [2], pp.247ff). Actually, intersection schemes can be considered as a generalization of uniform oriented matroids of rank 3, which becomes clear from Bokowski's axiomatization of oriented matroids in terms of hyperline sequences (cf. [4], p.576). The latter correspond to the lists of our intersection schemes. More exactly, uniform oriented matroids of rank 3 satisfy the two (additional) conditions:

(i) The $i$-th list of $A$ consists exactly of the elements $\in\{+1,-1, \ldots,+n,-n\} \backslash\{+i,-i\}$.

(ii) $\pm k$ occurs in position $j(1 \leq j \leq n-1)$ of a list in $A$ if and only if $\mp k$ occurs in position $j+n-1$.

In the following section we present a fundamental algorithm that shows that intersection schemes describe all combinatorial properties of arrangements of pseudocircles. This

algorithm will allow us to characterize ordinary and strict representability of intersection schemes in Sections 3 and 4.

\section{The Face Algorithm}

Identifying each face of the arrangement graph with its counterclockwise boundary walk, we can give for each intersection scheme of an arrangement a set of closed walks (cf. Definition 2.3) corresponding to the faces of the arrangement. We will see that the faces of an arrangement are determined by its intersection scheme. Given an oriented edge $e$ in the intersection scheme $A$ there is a unique face $f$ with $e$ on its boundary walk. In order to determine the faces of the arrangement it suffices to find for each directed edge $e$ the next edge $e^{\prime}$ on the boundary of $f$. Since two edges neighbored on the boundary of a face cannot be part of the same pseudocircle, the corresponding edges in the intersection scheme are placed in different rows. If the edge $e$ terminates in the crossing $(k, \pm j)$ the initial crossing of the next edge $e^{\prime}$ is $(j, \mp k)$. Thus, what remains to do is to determine the direction of $e^{\prime}$ in row $j$. Actually, we have to consider the four cases illustrated in 
a)

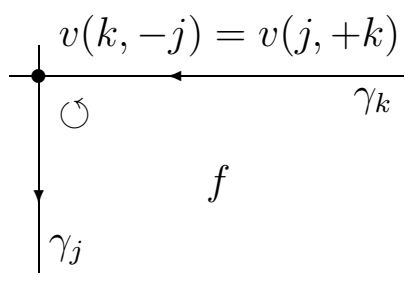

c)

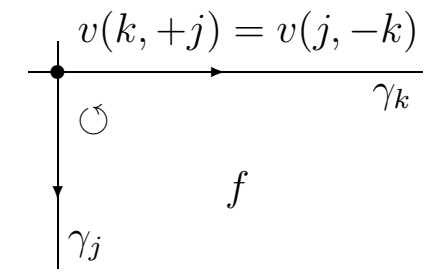

b)

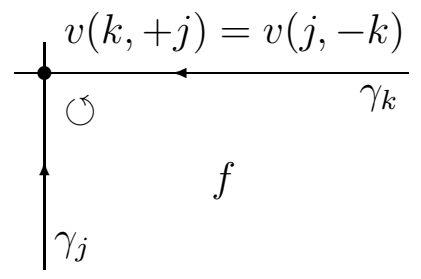

d)

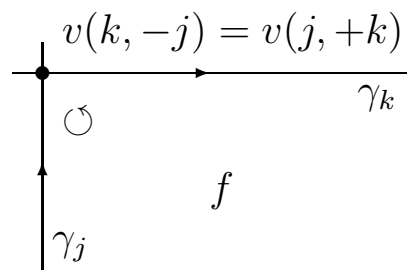

Figure 2: Boundaries of faces - all cases.

Figure 2. In case (a) we follow the orientation of $\gamma_{k}$ until we arrive at the crossing $(k,-j)$. Therefore, in the intersection scheme the edge $e$ is directed to the right, i.e. if $e$ starts in $v$ and terminates in $v^{\prime}$, the corresponding entries are ordered $v v^{\prime}$. The next edge $e^{\prime}$ has initial crossing $(j,+k)$ and according to the figure is also directed to the right. Working out all four cases the results can be summarized as in the following table.

\begin{tabular}{|c||c|c|c|}
\hline & first edge & second edge & crossings \\
\hline (a) & right & right & $v(k,-j)=v(j,+k)$ \\
(b) & right & left & $v(k,+j)=v(j,-k)$ \\
(c) & left & right & $v(k,+j)=v(j,-k)$ \\
(d) & left & left & $v(k,-j)=v(j,+k)$ \\
\hline
\end{tabular}

We see that combining the information about the direction of the first edge with the corresponding crossing yields a rule for the direction of the second edge. If the entry in list $k$ has negative sign (or the entry in list $j$ positive sign), the directions of the two edges are the same, otherwise not. Thus, we can give an algorithm that finds all faces of a given arrangement from its intersection scheme. A similar algorithm for rotation systems of a graph can be found in Gross, Tucker [10], p.114f.

\section{Face algorithm}

1. Choose an arbitrary (directed) edge in the scheme. Let $k$ be the list containing this edge and $v(k, \pm j)$ the terminal vertex.

2. The initial vertex of the next edge is $v(j, \mp k)$.

3. Determine the direction of the next edge by the sign of the entry $\mp k$ in list $j$ and the direction of the preceding edge according to the table above. 
4. Go to step 2 until a (directed) edge occurs which has already been visited before. If this happens, the obtained closed walk corresponds to a face of the arrangement.

5. Repeat the algorithm with a (directed) edge that does not occur in any of the closed walks found so far.

Note that in the resulting closed walks it may happen that a vertex is visited more than once or that an (undirected) edge occurs twice.

We may now apply this algorithm to an arbitrary (abstract) intersection scheme.

Proposition 3.1. For each intersection scheme, the face algorithm finally returns in step 4 to the edge it started with in step 1.

Proof. Let us assume that the algorithm arrives at a previously visited edge $e$ with initial crossing $(i, \pm k)$ that is not the edge it started with. Let $e_{1}, e_{2}$ be the two edges preceding $e$ in the course of the face algorithm. Then $e_{1}$ and $e_{2}$ must have the same terminal crossing $(k, \mp i)$, i.e. $e_{1}$ and $e_{2}$ are in list $k$ oppositely directed towards the same crossing. According to the algorithm the next edge is determined by the direction of the current edge and the sign of its terminal crossing, so that $e_{1}$ and $e_{2}$ cannot both be followed by $e$, which contradicts our initial assumption.

By Proposition 3.1 we immediately get

Theorem 3.2. Applying the face algorithm to an arbitrary intersection scheme results in a set of closed walks, such that each directed edge of the scheme occurs in exactly one of these walks.

We have seen that the arrangement graph as well as the face set of the arrangement can be derived from the (up to permutation of the labels) unique intersection scheme. Thus intersection schemes provide us with a good definition of combinatorial isomorphy for arrangements of pseudocircles.

Definition 3.3. Two arrangements $\Gamma, \Gamma^{\prime}$ are isomorphic if there is a labelling of the pseudocircles in $\Gamma$ and $\Gamma^{\prime}$, respectively, such that $\Gamma$ and $\Gamma^{\prime}$ have the same intersection scheme.

\section{Topological Representations of Intersection Schemes}

\subsection{Surfaces from Intersection Schemes}

Given an intersection scheme $A$, we can derive a (connected) graph $(V(A), E(A))$ and (applying the face algorithm) a set $F(A)$ of closed walks in $(V(A), E(A)$ ). The resulting structure $K(A)=(V(A), E(A), F(A))$ is a (combinatorial) cell complex (cf. [16] or the original [17]). The aim of this and the following section is to show that each cell complex obtained from an intersection scheme corresponds to an arrangement of pseudocircles on some closed orientable surface. To this end we are going to use the following definition (cf. [3], p.58 and [9], p.56): 
Definition 4.1. A cell complex $K=(V, E, F)$ is called a closed orientable surface if the following conditions are satisfied.

(i) The graph $(V, E)$ is connected.

(ii) Faces in $F$ have common vertices only to the extent required by the common edges, that is, the faces $f_{1}, f_{2}, \ldots f_{k}$ incident with a given vertex $v$ can always be arranged in a cycle $f_{i_{1}}, f_{i_{2}}, \ldots f_{i_{k}}, f_{i_{1}}$ such that two consecutive faces have an edge incident with $v$ in common. Here the faces are counted according to the number of incidences with $v$ so that a face may occur several times among the $f_{1}, f_{2}, \ldots f_{k}$.

(iii) Each edge in $E$ occurs exactly twice on the boundary of faces in $F$.

(iv) All faces in $F$ can be oriented such that neighbored faces induce opposite directions on their common edge.

Theorem 4.2. Cell complexes $K(A)$ derived from intersection schemes $A$ are always closed orientable surfaces.

Proof. First note that (i) holds due to the assumption that $A$ is connected (condition (iv) of Definition 2.4), while (iii) and (iv) are satisfied by the definition of the face algorithm. To see that condition (ii) is fulfilled as well, let $v$ be an arbitrary vertex and $e_{1}, e_{2}, e_{3}, e_{4}$ the four edges incident with $v$, so that $e_{1}, e_{3}$ belong to the same list and $e_{2}, e_{4}$ are placed in some other list. Starting the face algorithm with the edge $e_{1}$ (directed towards $v$ ), the next edge is either $e_{2}$ or $e_{4}$. On the other hand, if we apply the algorithm to the edge $e_{3}$ (directed towards $v$ ), the next edge is $e_{4}$ or $e_{2}$ (depending on the outcome for $e_{1}$ above). Considering all possible cases, one easily sees that the situation is as shown in Figure 3 (possibly after renaming the edges $e_{2}$ and $e_{4}$ or reversing the order of the edges, respectively). Thus, for each vertex $v$ there are four faces incident with $v$ (counted

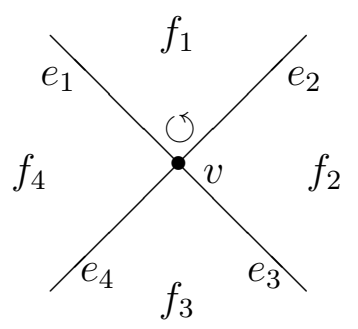

Figure 3: Edges and faces incident with $v$.

according to the number of incidences) which can be ordered such that (ii) is satisfied.

\subsection{Representations on Closed Surfaces and in the Plane}

Theorem 4.2 is the foundation for the characterization of representability of intersection schemes. It guarantees that the cell complex $K(A)=(V(A), E(A), F(A))$ is a closed 
orientable surface $\mathcal{S}$ so that we can interpret $K(A)$ as cellular embedding of the graph $(V(A), E(A))$ in $\mathcal{S}$ (i.e. an embedding with all faces homeomorphic to an open disc).

The following theorem establishes a one-to-one correspondence between intersection schemes and equivalence classes (with respect to isomorphy) of arrangements of pseudocircles on closed orientable surfaces.

Theorem 4.3. Every intersection scheme is representable on some closed orientable surface. More exactly, an intersection scheme $A$ is representable on a closed orientable surface of genus $g$ if and only if the Euler characteristic $\chi(K(A)) \geq 2-2 g$.

Proof. If $A$ is representable on a closed orientable surface of genus $g$ then clearly the Euler characteristic $\chi(K(A)) \geq 2-2 g$.

Now, suppose that $A$ is an intersection scheme for which the condition holds. Obviously, if an arrangement $\Gamma$ can be embedded in a surface of genus $g$, this also holds for surfaces with genus $>g$. Thus, we may assume without loss of generality that $\chi(K(A))=2-2 g$ so that $K(A)$ corresponds to an embedding of a graph in a surface $\mathcal{S}_{g}$ of genus $g$. Using the coloring algorithm of Section 2 we show that this graph is the arrangement graph of an arrangement of pseudocircles. From the observations made in the proof of Theorem 4.2 (cf. also Figure 3) it follows that if a vertex $v$ is incident with the edges $e_{1}, e_{2}, e_{3}, e_{4}$ (in clockwise order), then the edges $e_{1}, e_{3}$ are in the same list of $A$, while $e_{2}, e_{4}$ are placed in some other list. Hence the edge coloring algorithm colors two edges $e, e^{\prime}$ with the same color if and only if $e$ and $e^{\prime}$ are placed in the same list of $A$. Since all edges of an arbitrary list of $A$ form a cycle graph, we may conclude by Proposition 2.2 that the embedding of $K(A)$ in $\mathcal{S}_{g}$ is the arrangement graph of an arrangement $\Gamma$. Finally, note that each pair of pseudocircles in $\Gamma$ cannot have more than two intersection points with each other, since in each list $i$ there are at most two entries $\pm j$.

For representability in the plane we need an additional condition.

Definition 4.4. A crossing $\varepsilon$ is said to be within $i$ if there is a walk between the crossing $\varepsilon$ and another crossing $\varepsilon^{\prime}$ such that:

(i) The entry corresponding to $\varepsilon^{\prime}$ is placed strictly between entries $+i$ and $-i$ (in that order).

(ii) The walk does not contain entries $+i$ or $-i$.

In the case of generalized intersection schemes we obviously demand in (i) that the entry corresponding to $\varepsilon^{\prime}$ is placed between entries $+i^{\left(\ell_{1}\right)}$ and $-i^{\left(\ell_{2}\right)}$ such that no entry between $+i^{\left(\ell_{1}\right)}$ and $-i^{\left(\ell_{2}\right)}$ is of the form $-i^{(\ell)}$. In intersection schemes of strict arrangements a crossing is within $i$ if and only if the corresponding vertex is in the interior of $\gamma_{i}$.

Theorem 4.5. An intersection scheme $A$ is representable in the plane if and only if the following conditions hold:

(i) The Euler characteristic $\chi(K(A))=2$. 
(ii) There is a crossing in $A$ which is not within any $i$.

Proof. The crossing in condition (ii) corresponds to a vertex that is not contained in the interior of any pseudocircle. Clearly such a vertex appears in any arrangement in the plane.

On the other hand, let $A$ be an intersection scheme with (i) and (ii) satisfied. By Theorem 4.3, $A$ is representable on the sphere. Since (ii) guarantees the existence of a vertex that is not inside any pseudocircle, there must be a face $f$ with the same property as well. We use stereographic projection from an arbitrary point in the interior of $f$ to map the arrangement from the sphere into the plane. Note that in this way the interior of each pseudocircle is mapped onto a bounded region in the plane so that all pseudocircles in the plane are oriented counterclockwise.

\section{Consistency and Strict Representations}

On surfaces of genus $>0$ the question of strict representability of intersection schemes arises. Two non-separating pseudocircles $\gamma_{i}, \gamma_{j}$ may have only a single intersection point or they may cross each other so that both intersection points correspond to entries of the form $+j($ or $-j)$ in list $i$. In these cases the intersection scheme of the arrangement is not pure. However, pureness is not a sufficient condition for strict representability.

Definition 5.1. A pure intersection scheme is said to be inconsistent if there is a $j \in$ $\{1,2, \ldots, n\}$ and a walk between two entries $\varepsilon, \varepsilon^{\prime}$ such that:

(i) $\varepsilon$ lies strictly between $+j$ and $-j$ (in that order).

(ii) $\varepsilon^{\prime}$ lies strictly between $-j$ and $+j$ (in that order).

(iii) The walk does not contain any entries $\pm j$.

If $A$ is pure but not inconsistent, $A$ is said to be consistent. In the case of generalized intersection schemes (i) and (ii) have to be adapted like condition (i) in Definition 4.4.

Thus representable inconsistent intersection schemes do not allow a distinction between interior and exterior for the pseudocircle $j$. Hence inconsistent intersection schemes are never strictly representable. The following theorem shows that the converse holds as well.

Theorem 5.2. Every consistent intersection scheme is strictly representable.

For the proof of Theorem 5.2 we need the following lemma.

Lemma 5.3. Let $G=(V, E)$ be a graph that is cellularly embedded in $\mathcal{S}_{g}$ with $g>0$. Let $\mathcal{C}_{1}$ be a non-separating cycle in $G$. Then there is a second non-separating cycle $\mathcal{C}_{2}$ in $G$, such that the graph $G^{\prime}$ consisting of the vertices and edges of $\mathcal{C}_{1}$ and $\mathcal{C}_{2}$ is connected and the induced embedding of $G^{\prime}$ in $\mathcal{S}_{g}$ has a single face. 
Proof. Let us first consider the case $g=1$ where $\mathcal{S}_{g}$ is homeomorphic to a torus. The induced embedding of $\mathcal{C}_{1}$ in $\mathcal{S}_{g}$ has a single face homemorphic to an (open) cylinder. Since the embedding of $G$ in $\mathcal{S}_{g}$ is supposed to be cellular there must be a simple path $P$ in $G$ connecting the "left side" of $\mathcal{C}_{1}$ with its "right side". Thus the edges and vertices in $P$ together with a suitable (possibly empty) subgraph of $\mathcal{C}_{1}$ form a cycle $\mathcal{C}_{2}$ in $G$ with the desired properties. If $g>1$ then $\mathcal{S}_{g}$ is homeomorphic to a connected sum of $g$ tori and an analogous argument applies.

Proof of Theorem 5.2. Suppose $A$ is a consistent (and hence pure) intersection scheme that is not strictly representable. Let $\Gamma$ be an arrangement with intersection scheme $A$ cellularly embedded in some surface $\mathcal{S}_{g}(g>0)$ (obtained via the face algorithm). Let $\gamma_{i}$ be a non-separating pseudocircle $\in \Gamma$. By Lemma 5.3 there must be another nonseparating cycle $\mathcal{C}$ in the arrangement graph such that the situation is as shown in one of the pictures of Figure $4 .^{2}$ Obviously, the part of the cycle $\mathcal{C}$ that does not lie on $\gamma_{i}$
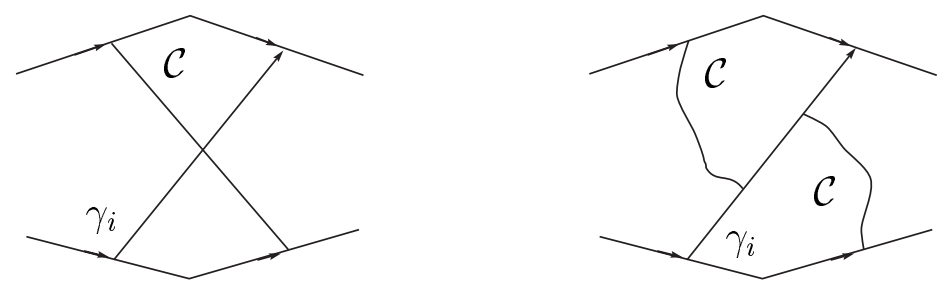

Figure 4: The non-separating pseudocircle $\gamma_{i}$ and the cycle $\mathcal{C}$.

cannot consist of edges belonging to a single pseudocircle $\gamma_{k} \in \Gamma$ : In the first case, $\gamma_{i} \cap \gamma_{k}$ would consist of a single element. In the second case, there are two vertices $\in \gamma_{i} \cap \gamma_{k}$ that correspond to an entry $+i$ (or $-i$ ) in the $k$-th list of $A$. In both cases $A$ violates the condition for pureness. Thus, there are edges of at least two different pseudocircles in $\mathcal{C}$ and the situation is as shown in one of the two pictures of Figure 5.
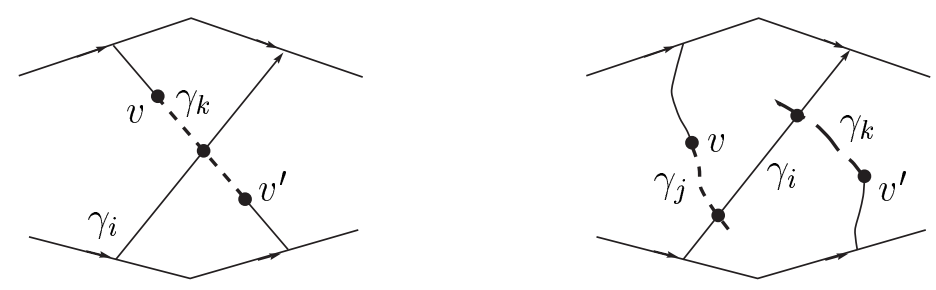

Figure 5: Finding inconsistencies in the intersection scheme.

In the first case, by pureness the crossing in list $k$ corresponding to the vertex $v$ is placed between $+i$ and $-i$, while $v^{\prime}$ is placed between $-i$ and $+i$. (Note that this is true independent of the orientation of $\gamma_{k}$ in Figure 5.) Since these two crossings can be

\footnotetext{
${ }^{2}$ The figures are based on the familiar representation of a closed surface of genus $g>0$ by a polygon with properly identified pairs of sides.
} 
connected by a walk without any entries $\pm i$ (cf. Figure 5$), A$ is inconsistent, contrary to our assumption.

The situation in the second case is similar. Again using pureness, the crossing in list $j$ corresponding to $v$ is placed between $+i$ and $-i$, while the crossing in list $k$ corresponding to $v^{\prime}$ is placed between $-i$ and $+i$ (again independent of the orientations of $\gamma_{j}$ and $\gamma_{k}$ in Figure 5). As in the previous case, we find a walk connecting these two crossings that does not contain any entries $\pm i$. (In the possible case where $v=v^{\prime}$ the walk degenerates to a single vertex.) Hence $A$ is inconsistent, which contradicts our assumption.

Theorem 5.2 immediately yields a one-to-one correspondence between consistent intersection schemes and strict arrangements of pseudocircles.

Theorem 5.4. An intersection scheme is strictly representable on some closed orientable surface if and only if it is consistent.

\section{Final Remarks}

Obviously the results of this paper can be used for computer aided enumeration of arrangements of pseudocircles. The face algorithm can easily be implemented and consistency may be checked computationally as well. For complete arrangements (i.e. arrangements where each two pseudocircles intersect in exactly two points) of three and four pseudocircles on the sphere this has been done in [15].

Bokowski et al. [6] used hyperline sequences to simplify the proof of the FolkmanLawrence topological representation theorem for arbitrary rank. Compared to the proof of the rank 3 case in [5] more sophisticated topological tools such as the generalized Schönflies theorem are needed. Thus, taking into account a bit more topology it might be interesting to try to generalize our results to higher dimensions. However, a priori it is not even clear how the face algorithm should be adapted to that case.

\section{References}

[1] P. K. Agarwal, Intersection and decomposition algorithms for planar arrangements, Cambridge University Press, Cambridge, 1991.

[2] A. Björner and M. Las Vergnas and B. Sturmfels and N. White and G. Ziegler, Oriented matroids, Encyclopedia of Mathematics and its Applications, Vol. 46, Cambridge University Press, 1999.

[3] D. W. Blackett, Elementary Topology. A Combinatorial and Algebraic Approach, Academic Press, New York, 1967.

[4] J. Bokowski, Oriented matroids, in P. M. Gruber and J. M. Wills, Handbook of Convex Geometry, pp. 555-602, Elsevier Science Publishers B.V., Amsterdam, 1993. 
[5] J. Bokowski, S. Mock, and I. Streinu, On the Folkman-Lawrence Topological Representation Theorem for Oriented Matroids of Rank 3, Europ. J. Combinatorics 22 (2001), 601-615.

[6] J. Bokowski, S. King, S. Mock, and I. Streinu, A Topological Representation Theorem for Oriented Matroids, to appear in Discrete Comput. Geom.

[7] K. L. Clarkson, H. Edelsbrunner, L. J. Guibas, M. Sharir, and E. Welzl, Combinatorial complexity bounds for arrangements of curves and spheres, Discrete Comput. Geom. 5 (1990), no. 2, 99-160.

[8] H. Edelsbrunner, L. Guibas, J. Pach, R. Pollack, R. Seidel, M. Sharir, Arrangements of curves in the plane-topology, combinatorics, and algorithms, Theoret. Comput. Sci. 92 (1992), no. 2, 319-336.

[9] P. J. Giblin, Graphs, Surfaces and Homology, Chapman and Hall, London, New York, 1981.

[10] J. L. Gross and T. W. Tucker, Topological Graph Theory, Wiley-Interscience, New York, 1987.

[11] B. Grünbaum, Venn Diagrams and Independent Families of Sets, Mathematics Magazine 48 (1975), 12-23.

[12] L. J. Guibas, M. Sharir, S. Sifrony, On the general motion-planning problem with two degrees of freedom, Discrete Comput. Geom. 4 (1989), no. 5, 491-521.

[13] C. Iwamoto and G. T. Toussaint, Finding hamiltonian circuits in arrangements of Jordan curves is NP-complete, Inform. Process. Lett. 52/4 (1994), 183-189.

[14] K. Kedem, R. Livné, J. Pach, M. Sharir, On the union of Jordan regions and collisionfree translational motion amidst polygonal obstacles, Discrete Comput. Geom. 1 (1986), no. 1, 59-71.

[15] R. Ortner, Arrangements of Pseudocircles, Ph.D. Dissertation, University of Salzburg, 2001.

[16] S. Rees and L. H. Soicher, An algorithmic approach to fundamental groups and covers of combinatorial cell complexes, J. Symbolic Computation 29/1 (2000), 59-77.

[17] K. Reidemeister, Einführung in die kombinatorische Topologie, Friedr. Vieweg \& Sohn, Braunschweig, 1951.

[18] M. Sharir, On $k$-sets in arrangements of curves and surfaces, Discrete Comput. Geom. 6 (1991), no. 6, 593-613.

[19] J. Snoeyink, J. Hershberger, Sweeping arrangements of curves, Discrete and computational geometry (New Brunswick, NJ, 1989/1990), 309-349, DIMACS Ser. Discrete Math. Theoret. Comput. Sci., 6, Amer. Math. Soc., Providence, RI, 1991. 